\title{
Characterization of an $N$-Acylated Glucagon-Like Peptide-1 Derivative by Electron Capture Dissociation
}

\author{
Kim F. Haselmann \\ Department of Chemistry, University of Southern Denmark, Odense, Denmark
}

\author{
Per F. Nielsen
}

Novo Nordisk A/S, Protein Science, Bagsværd, Denmark

\begin{abstract}
Roman A. Zubarev
Laboratory for Biological and Medical Mass Spectrometry, Uppsala Biomedical Center, Uppsala University, Uppsala, Sweden
\end{abstract}

An $N$-acylated glucagon-like peptide 1 derivative was characterized by Fourier transform ion cyclotron resonance mass spectrometry. Both electron capture dissociation (ECD) and sustained off-resonance irradiation collisionally activated dissociation (SORI-CAD) were employed. While ECD revealed full sequence coverage, site of modification, branching point, structure of the palmitoylated modification, SORI-CAD produced less complete and more ambiguous information attributable to facile losses of the fatty acid group from both parent and fragments. Thus, ECD showed a superior characterization performance over SORI-CAD in analysis of $\mathrm{N}$-acylated polypeptides. (J Am Soc Mass Spectrom 2005, 16, 548-552) (C) 2005 American Society for Mass Spectrometry

$\mathrm{T}$ The non-insulin-dependent diabetes mellitus also known as Type 2 diabetes is the most common form of diabetes. Type 2 diabetes has been considered a disease of overweight adults that develops starting in middle life, but now an increasing number of incidents are being found throughout the whole population. Therefore, Type 2 diabetes represents an increasing health challenge of the Western civilization. So far, this type of diabetes has to some extent been treated in obese persons through the indirect regulation of plasma glucose concentration. After the discovery of the glucagon-like peptides (GLP) [1], GLP-1 has received much attention because of its many useful features, although the complete physiological role in healthy humans and animals is not yet fully identified [2]. Among functions are the insulinotropic action [3], inhibition of gastric emptying and gastric acid secretion [4], stimulation of growth and proliferation of pancreatic $\beta$-cells [5], which are all highly desirable in regulating Type 2 diabetes. GLP-1 could therefore be of great

Published online February 25, 2005

Address reprint requests to Dr. K. F. Haselmann, Department of Chemistry, University of Southern Denmark, Campusvej 55, DK-5230 Odense, Denmark. E-mail: kfh@chem.sdu.dk value in the treatment of Type 2 diabetes and impaired glucose tolerance.

The full amino acid sequence of GLP-1 is ${ }^{1}$ HDEFERHAEGTFTSDVSSYLEGQAAKEFIAWLVKGRG ${ }^{37}$, whereas the active hormone is the truncated ${ }^{7} \mathrm{GLP}-^{36}$ amide that has $\sim 50 \%$ homology with glucagon. Both degradation by dipeptidyl peptidase IV causing inactivity [6] and renal clearance [7] are severe, making once daily administration impossible. These disadvantageous processes can, however, to some extent be circumvented by fatty acid derivatization that facilitates binding to serum albu$\min [8]$.

Mass spectrometry is an increasingly valuable tool used in chemistry, biochemistry, molecular biology, proteomics, and life sciences. The mass spectrometric characterization of compounds relies not only on measuring $m / z$, in which isomers are indistinguishable, but also on unimolecular dissociation in the gas phase. The observed ion fragment masses can then be reconstructed into the original molecule under investigation. Tandem mass spectrometry is thus a fast and powerful tool for compound characterization. Unimolecular fragmentation in the gas phase can be performed by employing several techniques, e.g., infrared multiphoton dissociation (IRMPD, [9]), black-body infrared radiative dissociation (BIRD, [10]), sustained off-resonance irradiation collisionally activated dissociation (SORI-CAD, 
[11]) and electron capture dissociation (ECD, [12]). The latter technique has shown promising advantages for characterizing polypeptides with labile modifications, e.g., phosphorylation [13, 14], O-glycosylation [15], Nglycosylation [16], $\gamma$-carboxylation [17], and $O$-acylation [18].

In the current study, an $N$-acylated polypeptide from GLP-1 [8] is characterized for the first time by electrospray ionization (ESI) Fourier-transform ion cyclotron resonance (FTICR) tandem mass spectrometry with ECD and SORI-CAD. The derivative is currently one of the best drug candidates for treatment of Type 2 diabetes via the GLP-1 receptor target.

\section{Experimental}

\section{Sample}

The $\gamma$-L-glutamoyl $\left(N^{\alpha}\right.$-hexadecanoyl)-R ${ }^{34}-{ }^{7}$ GLP- $1^{37}$ polypeptide (MW 3751) was synthesized in-house and was described elsewhere [8].

\section{Mass Spectrometry}

The polypeptide was dissolved in a standard electrospray mixture of water, methanol and acetic acid (49: $49: 2, \mathrm{v} / \mathrm{v})$ to the concentration of $10^{-5} \mathrm{M}$. A 3-5 $\mu \mathrm{l}$ aliquot was loaded into a metal-coated pulled-glass capillary (Proxeon, Odense, Denmark). Nano-electrospray ionization (nESI) using a hexapole-based interface (Analytica, Branford, MA) modified with a heated metal capillary was performed on a $4.7 \mathrm{~T}$ Ultima FT mass spectrometer from IonSpec (Irvine, CA). The electrospray-produced multiply charged ions were externally accumulated in the hexapole for $0.5 \mathrm{~s}$ and then transmitted to the ICR cell by RF-only quadrupolar ion guides. Capture of ions in an open-ended cylindrical cell with extra trapping plates was achieved by gated trapping. For ECD, the ions of interest were isolated by application to the excitation electrodes of a pre-programmed waveform. An indirectly heated dispenser cathode ca. $3 \mathrm{~mm}$ in diameter operated at $5 \mathrm{~V}$ and 1.18 A was employed [19]. During ECD, the electron-emitting surface was biased to $-1 \mathrm{~V}$ for $100-200 \mathrm{~ms}$. Between 100 and 200 acquisitions were accumulated and averaged. In Fourier transformation of the time domain, one zero-filling without apodization was performed. Mass spectra were peak-matched with respect to the parent ion peak. Collisionally activated dissociation (CAD) was performed in comparison employing sustained off-resonance irradiation (SORI). In-cell isolated parent ions were excited by a dipolar RF burst (10 $\mathrm{V}_{b-p}$ ) at a frequency $\sim 2 \mathrm{kHz}$ lower than the resonance frequency of the parent ion for $500 \mathrm{~ms}$ while increasing the pressure. A pulsed valve was opened for $2 \mathrm{~ms}$ that was connected to a reservoir filled with $\mathrm{N}_{2}$ gas at a pressure of 21 torr. Twenty acquisitions were accumulated and averaged.

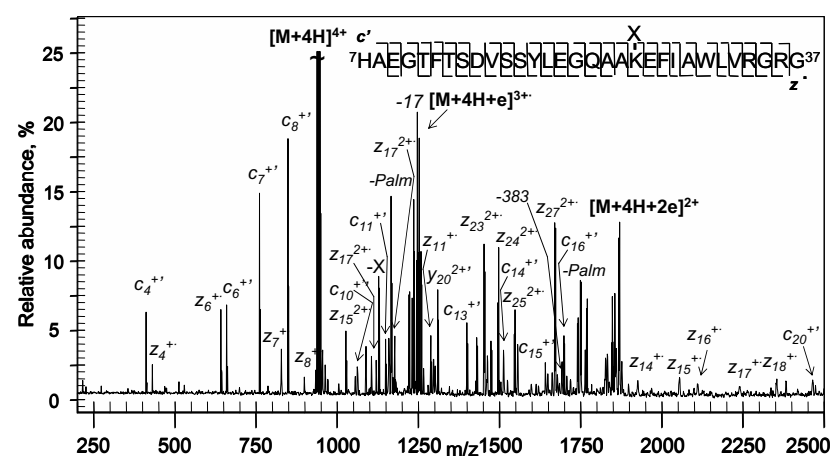

Figure 1. ECD MS/MS spectrum of the quadruply charged $(\mathrm{m} / \mathrm{z}$ 938) $\gamma$-L-glutamyol $\left(N^{\alpha}\right.$-hexadecanoyl) modified $\mathrm{Arg}^{34}{ }_{-}{ }^{7} \mathrm{GLP}-1^{37}$ derivative. The inter residue cleavage sites are shown at the upper right corner. The $\gamma$-L-glutamyol $\left(N^{\alpha}\right.$-hexadecanoyl) modification is shown as $\mathrm{X}$ and $-\mathrm{X}$ represents the complete loss of the modification leaving the intact polypeptide. Loss of part of the modification (palmitoyl amide) is shown as: - Palm (200 scans).

\section{Results and Discussion}

In the electrospray spectrum (not shown) of the ${ }^{7}$ GLP- $1{ }^{37}$ derivative, abundant multiply charged species of $4+$ and $3+$ were observed that corresponded to the molecular mass of $3748.95 \mathrm{Da}$. Compared to the nonderivatized ${ }^{7} \mathrm{GLP}-1^{37}$ peptide (3353.67 Da) this was a mass difference of 395.28 Da. The highest and most abundant charge state $(4+)$, reflecting the presence of four basic residues in the sequence, was selected for electron capture dissociation. In the ECD spectrum of the derivative (Figure 1), full sequence coverage was obtained except the cleavage between the first two amino acids at the N-terminal side (97\%), as $c_{1}^{+\prime}$ (here, the $c^{\prime}$ nomenclature [20] is used to indicate the incorporation of a hydrogen atom in the $c$-fragment) was outside the recorded mass range and a $z_{30}$-fragment was not observed (neither as $2+$ nor as $3+$ ). However, the mass of the ${c_{2}}^{+\prime}$ ion unambiguously corresponding to the mass of the amino acid pair histidine and alanine, was therefore a positive identification of these two amino acids, but not the order. In the derivatized polypeptide, arginine was correctly assigned at the amino acid position 34 , which differs from the original ${ }^{7} \mathrm{GLP}-1^{37}$ polypeptide that had a lysine residue at that position. This leaves the remaining derivatized polypeptide with a modification of 367.27 Da. From the $c^{\prime}$-ion sequence pattern the difference between $c_{19}{ }^{+\prime}$ and $c_{20}{ }^{+\prime}$ corresponded to the mass of a lysine amino acid residue and the modification, which permitted the localization of the modification to lysine ${ }^{26}$. Likewise, the difference between the $z_{11}{ }^{+\cdot}$ and the $z_{12}{ }^{+\cdot}$ ions was $495.31 \mathrm{Da}$ that also matched the modified lysine $\mathrm{e}^{26}$.

The structure of the modification itself was revealed from the regions near the charge-reduced species [M + $4 \mathrm{H}+\mathrm{e}]^{3+\cdot}$ (also known as the $(\mathrm{M} \cdot-\mathrm{X})$ region [21]) and $[\mathrm{M}+4 \mathrm{H}+2 \mathrm{e}]^{2+}$. A loss of 255.27 Da from the $[\mathrm{M}+4 \mathrm{H}$ $+\mathrm{e}]^{3+\cdot}$ species indicated loss of palmitoyl amide (255.256 Da, see Scheme 1). This loss was also seen from the doubly-reduced species, $[\mathrm{M}+4 \mathrm{H}+2 \mathrm{e}]^{2+}$. The C16:0 fatty acyl chain was linked to the $N^{\alpha}$-amine group 


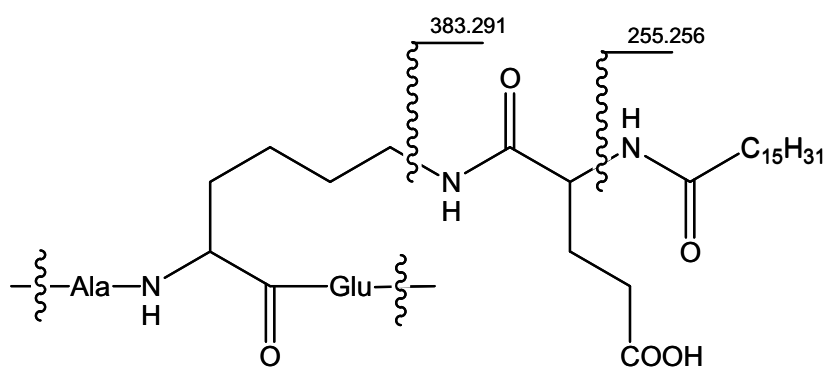

Scheme 1

of an amino acid residue through an amide bond ( $N$-acyl modified). The $\mathrm{C}^{\alpha}-\mathrm{N}$ bond between the palmitoyl and the glutamoyl residue was cleaved in ECD analogous to an equivalent peptide backbone bond, i.e., hydrogen atom transfer to the proton solvating carbonyl oxygen upon electron capture, followed by a radical site initiated $\alpha$-cleavage to form a $c^{\prime}$-type neutral fragment, which in this case had the same mass as palmitoyl amide (marked in Figure 1 as -Palm and seen in Scheme 1 as the 255 Da loss. This loss corresponds to the incorporation of a hydrogen atom). In an equivalent study by Guan [18] of $O$-acylated polypeptides, a loss of the full fatty acid chain was observed as well. The second part of the modification that linked the palmitoyl moiety to the polypeptide chain was a glutamoyl residue, which was linked to the $\varepsilon$-amine group of lysine $^{26}$ through an amide bond (see Scheme 1). The $\mathrm{C}^{\varepsilon}-\mathrm{N}$ bond was observed to be cleaved in ECD (producing a $c^{\prime}$-type neutral fragment and a $z$-fragment ion in the same way as described above), which identified the amino acid residue correctly as glutamoyl (seen in Scheme 1 as the difference between 383 Da loss and the 255 Da loss). However, this cleavage was only observed as the $2+$ peak in the region of the doubly-reduced species $[\mathrm{M}+4 \mathrm{H}+2 \mathrm{e}]^{2+}$ in low abundance. This could be ascribed to the unfavorable formation of a primary radical at $C^{\varepsilon}$ in the lysine ${ }^{26}$ side chain. In comparison, Olsen et al. [22] studied electron capture dissociation of peptide nucleic acids, where backbone cleavage was far less abundant than for polypeptides. This was mainly ascribed to the higher proton affinities of the nucleobases. However, the different structure of the peptide nucleic acid backbone compared to polypeptides was perhaps another important aspect. The peptide nucleic acid backbone consists of $\mathrm{N}$-(2-aminoethyl)glycine units (not single $\alpha$-amino acid units), and upon hydrogen attachment of the carbonyl and $\alpha$-cleavage, $c^{\prime}$ and $z$-ions would be produced analogs to polypeptide backbone dissociation, but the $z$-ions were now primary radical cations, not secondary, and thus less abundant. Interestingly, in that study facile nucleobase loss was observed from $z \cdot$-ions, indicating further or concomitant reactions of the less stable radical cations. Further reactions of $z$-ions are usually rare in conventional ECD, but something that is a common feature in hot electron capture dissociation [20, 23]. Furthermore, in a recent study by Cooper et al. [24] ECD of $\varepsilon$-peptides gave no $c^{\prime}$ - or $z$-fragment ions within the $\varepsilon$-amino acid backbone chain, only $a \cdot-$ and $y^{\prime}$-fragment ions were observed. An isolated peptide bond unit seems to fragment differently from $\alpha$-peptide bond chains.

Another abundant fragment ion that corresponded to the full loss of the modification was observed from both the triply and the doubly (less abundant) protonated reduced charge states (marked in Figure 1 as $-X$ ). However, this fragment ion corresponded to a $b, y^{\prime}$-type cleavage producing the original nonmodified polypeptide. This could be explained by vibrational excitation of $[\mathrm{M}+3 \mathrm{H}]^{3+}$ species formed upon hydrogen desorption from charge-reduced species, which was observed to occur in ECD of small and medium-size polypeptides [25]. Interestingly, the full modification was lost, not only the palmitoyl moiety (having only one carbonyl group). Therefore, at least two carbonyl groups separated by three bonds (like $b_{2}$-fragment ions) were necessary for observing this loss. This emphasizes the need for forming an oxazolone structure [26]. In fact, a very weak signal was observed at $m / z 368$ corresponding to the charged oxazolone structure of the glutamoylpalmitoyl moiety (Scheme 2). In the ECD mass spectrum of the derivatized polypeptide, no $c^{\prime}$ - nor $z \cdot-$ sequence ions lost the $\gamma$-L-glutamyol $\left(N^{\alpha}\right.$-hexadecanoyl $)$ modification. Furthermore, Cooper et al. analyzed branched peptides [24] having a $N^{\varepsilon}$-triglycyl modification on a lysine residue: $\mathrm{AcA}_{3} \mathrm{~K}\left(\mathrm{G}_{3}\right) \mathrm{A}_{3}-\mathrm{NH}_{2}$. This peptide, in a similar manner, lost the full triglycyl modification as observed in the present study. In the mentioned study the loss of the full modification was ascribed as a $y^{\prime}$-ion fragment coming from the minor $a$, $y^{\prime}$-fragmentation pathway in ECD, supported by the observation of another $y^{\prime}$-ion fragment. Cooper et al. [24] also observed the cleavage of the modified lysine side chain at the $\alpha$-carbon atom; this is not observed in the present study. Interestingly, this loss was seen for a
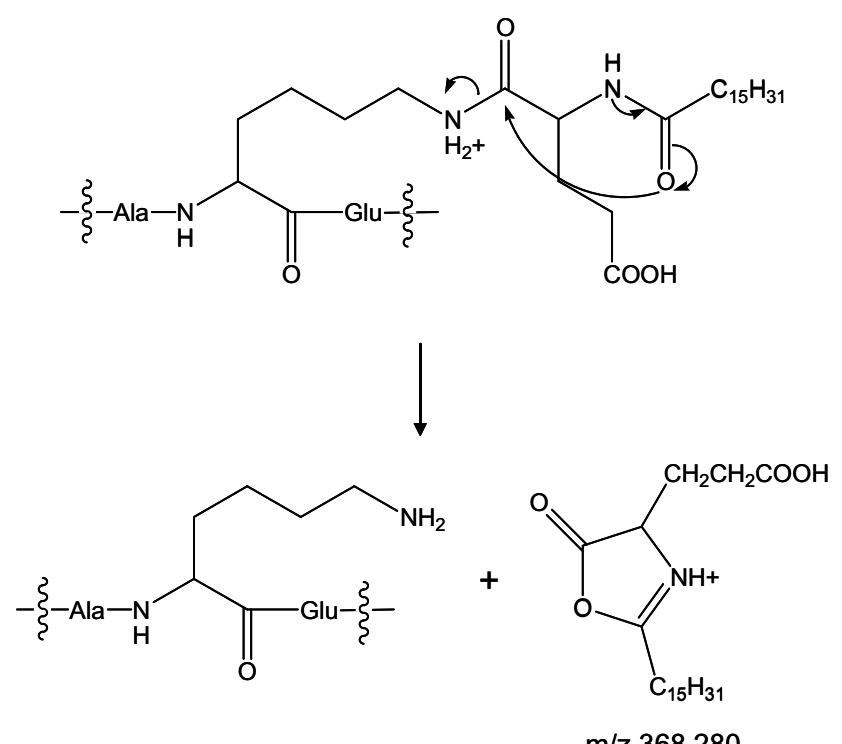

$\mathrm{m} / \mathrm{z} 368.280$

Scheme 2 


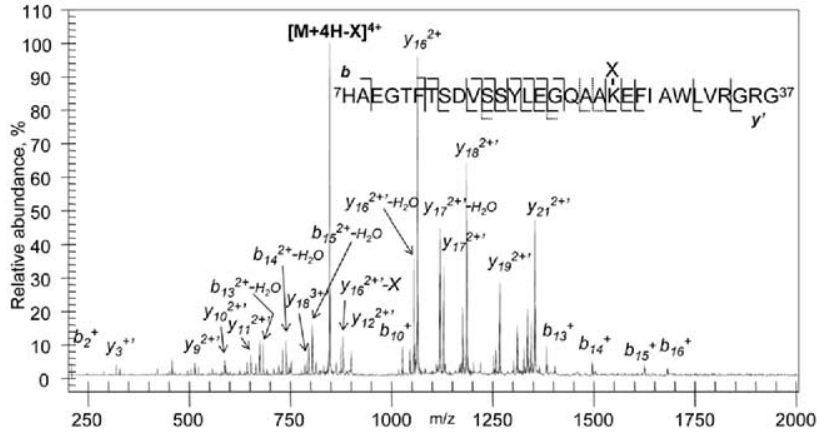

Figure 2. SORI-CAD MS/MS spectrum of the quadruply charged $\gamma$-L-glutamyol $\left(N^{\alpha}\right.$-hexadecanoyl) modified Arg $^{34}{ }^{7}$ GLP$1^{37}$ derivative. The inter residue cleavage sites are shown at the upper right corner (dashed lines are shown for loss of modification).öOtherösymbolsöareötheösameöasönöFigureöl.öDueötoötheöoff resonance excitation at $m / z 972$ for $500 \mathrm{~ms}(10 \mathrm{~V})$ a "blind spot" occurs in this area, where no peaks are seen (20 scans).

sodiated species and therefore the direct comparison is not possible.

For comparison, SORI-CAD was performed on the quadruply charged ${ }^{7} \mathrm{GLP}-1^{37}$ derivative (mass spectrum is shown in Figure 2). Here, $10 b$-ions and $15 y^{\prime}$ fragment ions were observed; cleaving 16 out of 30 possible inter-residue bonds (53\%). A total of four $y^{\prime}$-ions lost the full modification and of those, only two were observed without the modification making the modification site ambiguous. No $b$-ions higher than $b_{16}$ were observed. Nearly all major peaks were accompanied by satellite peaks equivalent to water losses (and in some cases, two consecutive water losses), from both $b$ and $y$-ions, which complicated the spectrum severely. This behavior was also observed in SORI-CAD of the $O$-acylated polypeptide ghrelin [18]. The base peak in the SORI-CAD spectrum was the quadruply charged nonmodified polypeptide chain, corresponding to the loss of the full modification. The branching site was indeed a labile site, which helps explain why this loss though much less prominent, was observed in ECD as well. Although upon hydrogen desorption the $[M+(n$ $-1) \mathrm{H}]^{(\mathrm{n}-1)+}$ species becomes vibrationally excited, the energy dissipated in the even-electron cation would only be enough for the lowest activation barrier to be overcome, if any. Typically, rearrangement reaction where bonds are both broken and formed would represent such low-energy activation barriers [27]. Furthermore, such reactions require, in general, a "tight" transition complex to be formed (e.g., five-member rings) that would lower the rate constant. On the second-long timescales of the FTICR experiments, reactions occurring with even very low rates would be observed. The formation of oxazolone structures could be considered such a reaction.

In the SORI-CAD spectrum (Figure 2) the modification site and mass would have been correctly assigned as $\mathrm{N}$-acylated lysine residues $[28,29]$. However, only in the ECD spectrum would the two-part nature (glutamoyl- and palmitoyl-) of the modification have been successfully revealed.

\section{Conclusions}

An N-acyl modified GLP-1 polypeptide derivative was successfully analyzed and the modification site elucidated. The modification that contained amide bonds, produced common for ECD type cleavages, which assisted in characterizing the modification structure itself. This shows that ECD can not only elucidate the primary sequence and reveal the modification sites, but can also give information on the internal structure of the modification by means of specific radical-site initiated cleavages. Furthermore, the characterized polypeptide represented a branched polypeptide, the branching point of which was successfully determined as in a previous study [24].

\section{Acknowledgments}

The FTMS instrument was funded by the Instrument Center Program (grant 9700471) and related programs (grants SNF 9801448 and STVF 0001242).

\section{References}

1. Bell, G. I.; Sanchez-Pescador, R.; Laybourn, P. J.; Najarian, R.đC.đxonduplicationändळivergenceänđ̈heđHumanđ̈Preproglucagon Gene. Nature 1983, 304, 368-371.

2. Nauck, M. A. Is Glucagon-Like Peptide 1 an Incretin Hormone? Diabetologia 1999, 42, 373-379.

3. Nauck, M. A.; Heimesaat, M. M.; Ørskov, C.; Holst, J. J.; Ebert, R.; Creutzfeldt, W. Preserved Incritin Activity of GlucagonLike Peptide-1 [7-36 Amide] but not of Synthetic Human Gastric-Inhibitory Polypeptide in Patients with Type-2 Diabetes-mellitus. J. Clin. Invest. 1993, 91, 301-307.

4. Wettergren, A.; Schjoldager, B.; Mortensen, P. E.; Muhre, J.; Christiansen, J.; Holst, J. J. Trunctaed GLP-1 (Proglucagon 78-107-Amide) Inhibits Gastric and Pancreatic Functions in Man. Dig. Dis. Sci. 1993, 38, 665-673.

5. Edvell, A.; Lindström, P. Endocrinology 1999, 140, 778-783.

6. Deacon, C. F.; Johnsen, A. H.; Holst, J. J. Degradtion of Glucagon-Like Pepetide-1 by Human Plasma in Vitro Yields an N-terminally Truncated Peptide that is a Major Endogenous Metabolite in Vivo. J. Clin. Endocrinol. Metab. 1995, 80, 952-957.

7. Deacon, C. F.; Knudsen, L. B.; Madsen, K.; Wiberg, F. C.; Jacobsen, O.; Holst, J. J. Dipeptidyl Peptidase IV Resistant Analogs of Glucagon-Like Peptide-1 which have Extended Metabolic Stability and Improved Biological Activity. Diabetology 1998, 41, 271-278.

8. Knudsen, L. B.; Nielsen, P. F.; Huusfeldt, P. O.; Johansen, N. L.; Madsen, K.; Pedersen, F. Z.; Thøgersen, H.; Wilken, M.; Agersø, H. Potent Derivatives of Glucagon-Like Peptide-1 with Pharmacokinetic Properties Suitable for Once Daily Administration. J. Med. Chem. 2000, 43, 1664-1669.

9. Woodin, R. L.; Bromse, D. S.; Beachamp, J. L. Multiphoton Dissociation of Molecules with Low Power Continuous Wave Infrared Laser Radiation. J. Am. Chem. Soc. 1978, 100, 32483250.

10. Price, W. D.; Schnier, P. D.; William, E. R. Tandem Mass Spectrometry of Large Biomolecule Ions by Blackbody Infrared Radiative Dissociation. Anal. Chem. 1996, 68, 859-866. 
11. Gauthier, J. W.; Trautman, T. R.; Jacobson, D. B. Sustained Off-Resonance Irradiation for Collision-Activated Dissociation Involving Fourier-Transform Mass Spectrometry-CollisionActivated Dissociation Technique that Emulates Infrared Multiphoton Dissociation. Anal. Chim. Acta 1991, 246, 211-225.

12. Zubarev, R. A.; Kelleher, N. L.; McLafferty, F. W. Electron Capture Dissociation of Multiply Charged Proteins Cations. A Nonergodic Process. J. Am. Chem. Soc. 1998, 120, 3265-3266.

13. Stensballe, A.; Jensen, O. N.; Olsen, J. V.; Haselmann, K. F.; Zubarev, R. A. Electron Capture Dissociation of Singly and Multiply Phosphorylated Peptides. Rapid Commun. Mass Spectrom. 2000, 14, 1793-1800.

14. Shi, S. D. H.; Hemling, M. E.; Carr, S. A.; Horn, D. M.; Lindh, I.; McLafferty, F. W. Phosphopeptide/Phosphoprotein Mapping by Electron Capture Dissociation Mass Spectrometry. Anal Chem. 2001, 73, 19-22.

15. Mirgorodskaya, E.; Roepstorff, P.; Zubarev, R. A. Localization of O-Glycosylation Sites in Peptides by Electron Capture Dissociation in a Fourier-Transform Mass Spectromter. Anal. Chem. 1999, 71, 4431-4436.

16. Håkansson, K.; Cooper, H. J.; Emmett, M. R.; Costello, C. E.; Marshall, A. G.; Nilsson, C. L. Electron Capture Dissociation and Multiphoton Dissociation MS/MS of an N-Glycosylated Tryptic Peptide to Yield Complementary Sequence Information. Anal Chem. 2001, 73, 4530-4536.

17. Kelleher, N. L.; Zubarev, R. A.; Busch, K.; Furie, B.; Furie, B. C.; McLafferty, F. W.; Walsh, C. T. Localization of Labile Posttranslational Modifications by Electron Capture Dissociation: The Case of $\gamma$-Carboxyglutamic Acid. Anal. Chem. 1999, 71, $4250-4253$.

18. Guan, Z. Q. Identification and Localization of the Fatty Acid Modification in Ghrelin by Electron Capture Dissociation. J. Am. Soc. Mass Spectrom. 2002, 13, 1443-1447.

19. Tsybin, Y. O.; Hakansson, P.; Budnik, B. A.; Haselmann, K. F.; Kjeldsen, F.; Gorshkov, M.; Zubarev, R. A. Improved LowEnergy Electron Injection Systems for High Rate Electron Capture Dissociation in Fourier-transform Ion Cyclotron Resonance Mass Spectrometry. Rapid Commun. Mass Spectrom. 2001, 15, 1849-1854.

20. Kjeldsen, F.; Haselmann, K. F.; Budnik, B. A.; Jensen, F.; Zubarev, R. A. Dissociative Capture of Hot (3-13 eV) Electrons by Polypeptide Polycations: An Efficient Process Accompanied by Secondary Fragmentation. Chem. Phys. Lett. 2002, 356, 201-206.

21. Haselmann, K. F.; Budnik, B. A.; Kjeldsen, F.; Polfer, N. C.; Zubarev, R. A. Can the (M- - X) Region in Electron Capture Dissociation Provide Reliable Information on Amino Acid Composition of Polypeptides? Eur. J. Mass Spectrom. 2002, 8, 461-469.

22. Olsen, J. V.; Haselmann, K. F.; Nielsen, M. L.; Budnik, B. A.; Nielsen, P. E.; Zubarev, R. A. Comparison of Electron Capture Dissociation and Collisionally Activated Dissociation of Polycations of Peptide Nucleic Acids. Rapid Commun. Mass Spectrom. 2001, 15, 969-974.

23. Kjeldsen, F.; Zubarev, R. A. Secondary Losses via $\gamma$-Lactam Formation in Hot Electron Capture Dissociation: A Missing Link to Complete de Novo Sequencing of Proteins. J. Am. Chem. Soc. 2003, 125, 6628-6629.

24. Cooper, H. J.; Hudgins, R. R.; Marshall, A. G. Electron Capture Dissociation Fourier-Transform Ion Cyclotron Resonance Mass Spectrometry of Cyclodepsipeptide, Branched Peptides, and $\varepsilon$-Peptides. Int. J. Mass Spectrom. 2004, 234, 23-35.

25. Zubarev, R. A.; Horn, D. M.; Fridriksson, E. K.; Kelleher, N. L.; Kruger, N. A.; Lewis, M. A.; Carpenter, B. K.; McLafferty, F. W. Electron Capture Dissociation for Structural Characterization of Multiply Charged Protein Cations. Anal. Chem. 2000, 72, 563-573.

26. Yalcin, T.; Khouw, C.; Csizmadia, I. G.; Peterson, M. R.; Harrison, A. G. Why are B Ions Stable Species in Peptide Spectra? J. Am. Soc. Mass Spectrom. 1995, 6, 1165-1174.

27. McLafferty, F. W.; Turecek, F. Interprtation of Mass Spectra, 4th ed.; University Science Books: Mill Valley, CA, 1996, pp 115-118.

28. Hackett, M.; Guo, L.; Shabanowitz, J.; Hunt, D. F.; Hewlett, E. L. Internal Lysine Palmitoylation in Adenylate Cyclase Toxin from Bordetella pertussis. Science 1994, 266, 433-435.

29. Havlicek, V.; Higgins, L. A.; Chen, W. B.; Halada, P.; Sebo, P.; Sakamoto, H.; Hackett M. Mass Spectrometric Analysis of Recombinant Adenylate Cyclase Toxin from Bordetella pertussis Strain 18323/pHSP9. J. Mass Spectrom. 2001, 36, 384-391. 